\title{
Cinco consideraciones en la composición arquitectónica de la vivienda social vertical en Aguascalientes [México] por el estilo de vida actual
}

DOI: $10.20396 /$ labore.v13i0.8653214

Submetido 20 ago. 2018

Aceito 19 der. 2018.

Publicado 03 jul. 2019.

\author{
Mauricio Ruiz Morales \\ $<$ https://orcid.org/0000-0003-0309-9694>
}

RESUMEN

La vivienda social debe de responder a las necesidades de la población de manera integral, considerando aspectos sociales, económicos, culturales y simbólicos, debido a que en ésta, se desarrollan diferentes actividades que ayudan a la estructuración social y simbólica de los individuos en sociedad. En el desarrollo de la edificación de vivienda social en vertical, los diseños deben de estar dirigidos hacia un adecuado habitar, fomentando las condiciones necesarias para ser una plataforma adecuada para el desarrollo comunitario. Por el contrario, en gran parte del territorio mexicano, en la actualidad, la producción de vivienda social tiene una respuesta más orientada a atender aspectos económicos del mercado de la producción de la edificación, que a las necesidades económicas y sociales de la población en condiciones de pobreza y vulnerabilidad en México. Hay una necesidad imperante en reflexionar acerca de la forma del uso de las viviendas sociales en vertical, así que, dentro del presente artículo, se muestran ciertas consideraciones (cinco aspectos) acerca del perfil de los beneficiarios de la vivienda social en vertical, que generan tensiones en la forma y estilo de habitar las viviendas. Para cubrir los fines de la investigación, se analizó un caso en la ciudad de Aguascalientes. La propuesta del presente escrito, tiene la intención de ser una herramienta reflexiva para tratar de encontrar posibilidades al momento de desarrollar complejos habitacionales dirigidos a la vivienda social en vertical.

PALABRAS CLAVE

Desarrollo del Hábitat Sostenible. Vivienda social. Aguascalientes. Pobreza. Línea de bienestar.

\section{Five considerations in the architectural composition of vertical social housing in Aguascalientes [Mexico] for the current lifestyle}

\begin{abstract}
Social housing must respond to the population and their needs in a comprehensive manner. Social, economic, cultural and symbolic aspects have to be considered, because in the homes, different activities are developed that help to consolidate the individuals in society with a social and symbolic approach. In the development of vertical social housing construction, the designs should be directed towards the way of living, as well as promoting conditions for community development. In contrast, in much of the Mexican territory, at present, the production of social housing has an answer to address economic aspects of the construction market, instead of meeting the economic and social needs of the population in conditions of poverty and vulnerability. There is a prevailing need to reflect on the vertical use of social housing, so, within the present article, there are certain considerations (five aspects) about the profile of the beneficiaries of vertical social housing, which generate tensions in the way and style of inhabiting houses. To cover the purposes of the investigation, a case was analyzed in the city of Aguascalientes. The proposal of this writing, is intended to be a reflective tool to try to find possibilities when developing housing complexes aimed at vertical social housing.
\end{abstract}

KEYWORDS

Development of Sustainable Habitat, social housing, Aguascalientes, poverty, welfare line. 


\section{Introducción}

Existen un par de preguntas fundamentales para plantear un proyecto arquitectónico de una vivienda social: ¿qué significa la casa para la persona que la va a habitar? y ¿qué se puede construir con los recursos destinados para la edificación, que cubra las expectativas del significado de la vivienda (o se aproxime a cubrirlas)?

Con el primer acercamiento, la primera pregunta parece tener un significado simple, directo y hasta cierto punto obvio. Sin embargo, al buscar una aproximación del significado de habitar desde la hermenéutica, la respuesta toma una dimensión mucho más estructurada dentro del campo de lo complejo'1.

Para aproximarnos a la construcción de una respuesta a la pregunta planteada, es necesario poner ciertas cuestiones en orden para que, paulatinamente podamos adentrarnos en el punto central del tema que se aborda en el presente artículo, ¿cuáles son las consideraciones básicas del estilo de vida de los habitantes de vivienda social vertical en la composición arquitectónica a partir de una correcta lectura del programa arquitectónico o del planteamiento de necesidades iniciales?

Existen diferentes condiciones que se estiman necesarias al momento de diseñar una vivienda. Algunas se consideran dentro de la norma por ser las medidas mínimas de una casa al responder a cuestiones antropométricas; sin embargo, la disposición para el funcionamiento de una vivienda en términos de su operación, se plantea necesario considerar características a partir del estilo de vida que se supone que deben de desempeñar los habitantes al momento de interactuar con la vivienda en el plano físico, es decir, entre los muros, pisos y techos como un contenedor para el desarrollo de ciertas actividades humanas.

\section{Desarrollo}

La producción de la vivienda social, desde el punto de vista de la hermeneusis y consecuentemente de la interpretación de las prácticas humanas, produce diferentes connotaciones para los diferentes tipos de observadores a partir de las composiciones de una edificación y su contexto². Así, una casa tiene una dimensión diferente para cada sector sociocultural en el sentido del estilo de vida con el que se utiliza en la vivienda.

Para ejemplificar lo anterior, podemos preguntar a personas con diferentes características acerca de su estructura socioeconómica y la respuesta, podrá tener validez sólo desde el punto en el que se emite un criterio determinado por el usuario, es decir, una pregunta tan simple como: ¿es correcto tener televisiones dentro de las recámaras?, genera una diversidad de posturas que vienen asociadas directamente al potencial de las actividades realizables dentro de la vivienda, junto al estilo de vida y prácticas sociales del que habita en la casa. Así, alguien que tiene el número suficiente de cuartos para destinar uno, como sala de televisión, puede preferir no equipar las habitaciones para dormir con televisión porque puede destinar un cuarto para ver televisión. Algunos de los posibles argumentos pueden ser que las recámaras o habitaciones (para dormir) tienen un fin de descanso y además, puede argumentar que un cuarto de televisión tiene un fin recreativo y con características diferentes al destinado para dormir. Por el contrario, alguien que no tenga el número de habitaciones suficientes para tener un cuarto de televisión, tendrá mayor propensión a poner televisores dentro del cuarto para dormir, por la simple razón de no tener otro espacio para realizar la actividad de ver televisión.

Con lo anterior, se hace evidente que las prácticas sociales realizadas a través de una casa, tienen una relación estrecha con las cualidades físicas de la misma, además de tener una relación de significado cultural de cada sector socioeconómico.

La casa es un lugar en el que se desarrollan, normalmente, diferentes prácticas comunitarias dentro del núcleo familiar. Así, ésta juega un papel fundamental para que se produzca y reproduzca el individuo en términos sociales, económicos y culturales con la tendencia de aportar para la consolidación de una comunidad.

Retomando la pregunta inicial acerca del significado de la casa, podemos afirmar que el sentido que se le da a una casa tiene una relación directa entre las potencias físicas de la misma y el significado que le transfiere el individuo por la perspectiva social y cultural con el que observa la casa. Con otras palabras, el

\footnotetext{
1 "Complexus significa lo que está tejido junto; en efecto, hay complejidad cuando son inseparables los elementos diferentes que constituyen un todo (como el económico, el político, el sociológico, el psicológico, el afectivo, el mitológico) y que existe un tejido interdependiente, interactivo e interretroactivo entre el objeto de conocimiento y su contexto, las partes y el todo, el todo y las partes, las partes entre ellas. Por esto, la complejidad es la unión entre la unidady la multiplicidad" (Morin, 1999: 17).

2 Además, de la estructura propia de la vivienda, ésta se encuentra en un contexto en donde la densificación y uso mixto de los edificios deben de tener la potencia para conectar a los usuarios con los servicios; la localización de los edificios de vivienda sobrepuestos al trazado urbano debe de propiciar, para ser inclusiva y potencialmente sostenible, la generación de espacios públicos de convivencia para mejorar la experiencia y la calidad del entorno en donde las familias se desenvuelvan en un ambiente mucho mejor en el habitar.
} 
significado de una casa para el habitante, se estructura a partir de lo que éste quiere, puede, desea e interpreta desde su idiosincrasia, su estilo de vida y por sus prácticas sociales dentro de una estructura social y económica determinadas.

El significado de una vivienda, no es estático ni unidireccional, a pesar de que en la práctica, en el diseño arquitectónico de las viviendas, se presente una tendencia a organizar los componentes de una casa a partir del uso, técnicas de construcción comunes de la región, paradigmas sociales y culturales imperantes en un momento determinado.

Con respecto a la vivienda social, es importante mencionar que el término se ha acuñado para referir al tipo de vivienda con rango de precios económicos y que además, tiene la potencia de ser más accesible para la población del sector económicamente más vulnerable de la sociedad, porque normalmente, se oferta con apoyos monetarios en la modalidad de subsidios otorgados por el estado, una asociación sin fines de lucro o una combinación de los dos apoyos.

En el fondo, la vivienda social responde, al igual que cualquier tipo de vivienda, a cuestiones económicas, en términos de su producción y del mercado. Sin embargo, por el rango de costo con el que se edifica, hay una menor posibilidad de ampliar sus características físicas y funcionales, es decir, tiene que ser lo más elementalmente posible. Lo anterior, se explica, debido a que los metros cuadrados construidos y el costo de cada uno de ellos, tienen que ser muy limitados para ser competitivos, asequibles para el sector al que se dirigen y atractivos por el bajo costo; cualquier característica formal, que no sea simplificada en lo constructivo, puede implicar un incremento en el costo final y consecuentemente, excluir la vivienda del segmento al que se dirige.

Los diferentes niveles de gobierno se encuentran interesados e involucrados para el desarrollo de vivienda social en el país ${ }^{3}$. Ya sea con el desarrollo directo de vivienda a través de los diferentes organismos nacionales y estatales, con subsidios y apoyos financieros, o con una política mucho más sólida en términos de accesibilidad de la vivienda, se promueve la producción de vivienda con gran interés, porque como se mencionó, es un factor fundamental para el desarrollo de la población.

Para los involucrados en la producción de vivienda social y su evaluación, como son los sectores gubernamentales, universidades, grupos colegiados, centros e institutos, se torna indispensable considerar los componentes propios y los factores de la dinámica poblacional para conocer las aspiraciones y potencias del mismo sector. Lo anterior, para que, a través de la edificación, los habitantes de las viviendas del sector social, puedan potenciar sus capacidades para producir una comunidad en torno a su vivienda.

Aunado a lo anterior, la atención para el desarrollo de la vivienda social tiene que abordarse desde ciertos problemas identificados por potencial de compra, hasta la adaptabilidad del espacio a las necesidades de una familia por su número de miembros. Esto se torna de gran relevancia porque al estudiar la cobertura de necesidad de un complejo habitacional de vivienda social, es notorio que no hay una respuesta adecuada con las condiciones de la vivienda para todos los habitantes.

A la luz de lo anterior, se presentan cinco consideraciones que son pertinentes al momento de producir proyectos para mejorar la investigación, promoción, fomento e impulso de la vivienda social a partir de la atención de características específicas en la producción arquitectónica y en el uso de la vivienda:

1. Análisis de ingreso promedio del solicitante.

2. Subsidios disponibles para ser destinados a la población solicitante.

3. Número de habitantes por vivienda.

4. Cuál es la forma en que se usa el espacio y se dispone el mobiliario.

5. Cuál es la preferencia de espacios para crecimiento de la vivienda por uso, en caso de poder tener una ampliación la edificación.

Las consideraciones mencionadas son el producto de un estudio ${ }^{4}$ realizado en el Estado de Aguascalientes

\footnotetext{
${ }^{3}$ Es pertinente mencionar que la producción de la vivienda social se desenvuelve, dentro del ámbito de las empresas desarrolladoras de vivienda social, como una propuesta de negocio con ciertos riesgos asociados a la dinámica económica del sector social.

${ }^{4} \mathrm{La}$ investigación referida es un estudio de campo realizado con rigor científico. A partir de la entrega de 270 viviendas sociales en vertical, en modalidad de condominios séxtuples, en el fraccionamiento Morelos II, en Aguascalientes, el Instituto de Vivienda Social y Ordenamiento de la Propiedad (IVSOP), realizó diferentes estrategias para conocer mejor el sector que atiende y poder hacer mejores propuestas para los beneficiarios.
} 
para conocer ciertos factores con mayor tendencia a impactar en la habitabilidad de vivienda social en vertical, a partir de factores socioeconómicos y sociodemográficos del sector al que se dirige.

A continuación, se amplían las características de los aspectos mencionados como relevantes en el planteamiento de un proyecto de vivienda social. Para tener una referencia de las consideraciones necesarias para diseñar vivienda social aplicadas a un caso de estudio, se mencionan algunos resultados sobresalientes de la encuesta, anteriormente referida, realizada por el Instituto de Vivienda Social y Ordenamiento de la Propiedad del Estado de Aguascalientes (IVSOP). Dicha encuesta se aplicó a habitantes de 90 departamentos, de 270 totales entregados, del complejo de vivienda social en vertical, Morelos II ${ }^{5}$, terminado en 2017, en el Municipio de Aguascalientes. Los datos se han complementado con información de fuentes oficiales.

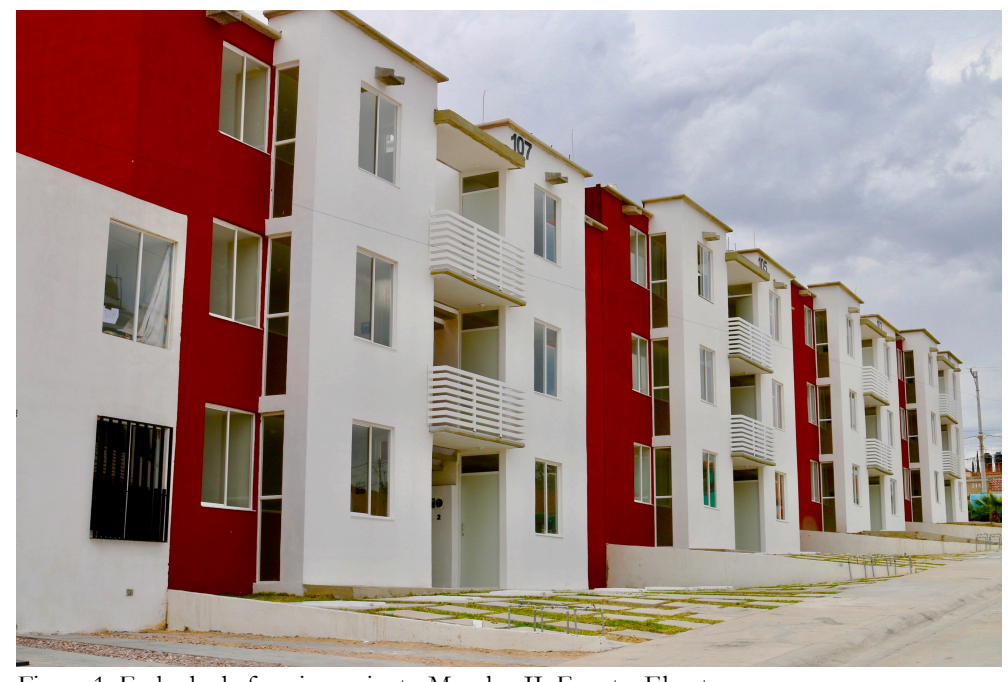

Figura 1. Fachada de fraccionamiento Morelos II. Fuente: El autor.

\section{Análisis de ingreso promedio del solicitante}

En la aspecto inicial que impide o da acceso a la adquisición de una vivienda social por parte de un solicitante con bajos ingresos, es la facilidad con la que este individuo puede obtener tanto los ahorros iniciales, como el crédito o hipoteca para poder adquirir un bien inmueble.

Si se considera que gran parte de la población solicitante no cuenta con un trabajo formal, y consecuentemente no tiene un apoyo por parte de algún organismo o institución para acceder a soluciones de vivienda, entonces, tanto el ingreso, como la potencia de ahorro y el potencial de crédito, son determinantes para la adquisición de una vivienda.

Es fundamental buscar que la solución de financiamiento de la vivienda no impacte de manera contundente en los ingresos familiares, ya que a pesar de que el costo de la vivienda es una condición para la construcción de la misma, estrategias innovadoras para soluciones financieras, apoyos y créditos pueden reducir la carga de endeudamiento familiar, y con esto el pago de la deuda crediticia.

El control en los gastos de una familia asociados a la vivienda por la ubicación de la misma, los servicios y transporte circundantes a ésta, es un aspecto fundamental, ya que el aumento en algún gasto prioritario, como el de la movilidad, escuela o costo mismo de la vivienda, implican una merma directa en la calidad de vida de la familia de escasos recursos. Por lo tanto, el análisis de la localización y movilidad de la vivienda con el apoyo de un estudio origen-destino de los beneficiarios en potencia, es una solución factible para la reducción de gastos asociados a la dinámica familiar.

Los créditos para una vivienda social tienden a comprometer al beneficiario entre 10 o 20 años. La facilidad del pago del crédito tiene que ver con el ingreso mensual de la familia y con los gastos que tienen la misma. Así, el estudio del ingreso familiar tiene gran sentido el objeto de buscar una menor presión financiera para los beneficiarios y un menor riesgo para las entidades financieras. Si se logra reducir el riesgo del crédito hay una tendencia en reducir el costo del préstamo y consecuentemente, hay una reducción en el costo total de la vivienda.

\footnotetext{
${ }^{5}$ El fraccionamiento, denominado como Reserva Morelos II, se localiza dentro de la Ciudad de Aguascalientes, al sur de la Avenida Siglo XXI, colindando con el fraccionamiento Morelos II. El proyecto de Morelos II, contempla 270 departamentos.
} 
Para conocer la presión de pago por parte del beneficiario, se pone en relieve que el ingreso de la mayoría de las familias no es el suficiente para cubrir el costo de la canasta básica alimentaria. De acuerdo con los cálculos del Consejo Nacional de Evaluación de la Política de Desarrollo Social (CONEVAL), el ingreso para cubrir el valor mensual de la canasta alimentaria urbana ${ }^{6}$ es de $\$ 1,476.96$ por persona, que equivale a $\$ 5,907.84$, para una familia de 4 miembros. El ingreso promedio de las familias evaluadas que habitan la vivienda social en vertical, tienen un ingreso entre los $\$ 3,900.00$ y $\$ 5,000.00$ (ver Figura 2) y el número promedio de habitantes es mayor a 4 integrantes. Por lo tanto, la mayoría de las familias evaluadas tienen una carencia o deficiencia en la alimentación.

Adicionalmente, es importante medir el estado de las familias de acuerdo con la línea de bienestar. Ésta, es la suma de la canasta alimentaria, adicionando los gastos de la canasta no alimentaria (educación, cuidados de salud, vestido, calzado, comunicación, transporte entre otros) y derivado del reporte más reciente (de junio de 2018), el valor mensual por persona para encontrarse arriba de la línea de bienestar es de $\$ 2,958.36$, para un núcleo de 4 personas asciende a $\$ 11,833.44$, aunque hay más de una tercera parte de departamentos con 5 habitantes o más. En este sentido, quedan más desfavorecidas las familias del estudio realizado.

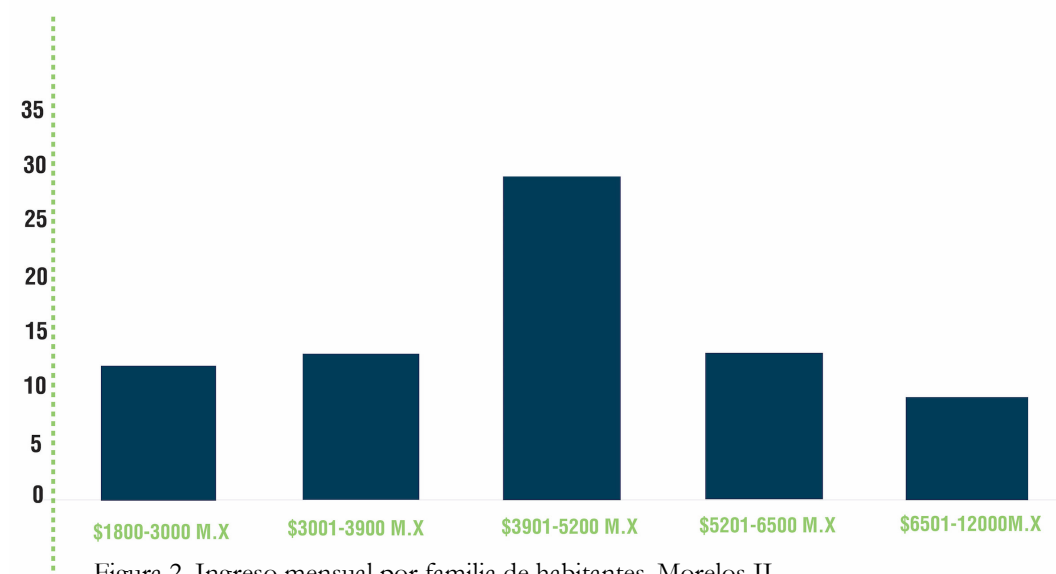

\section{Subsidios disponibles}

Una estrategia muy eficaz que aumenta las probabilidades para la adquisición de una vivienda social, para la población más vulnerable, son los subsidios de dependencias federales, estatales o municipales, ya que, estos se encuentran enfocados en mitigar el rezago habitacional, mejorar la calidad en los espacios de la vivienda, ampliar los metros construidos destinados a dormir, cocinar o aseo, así como, acceder a los servicios básicos de una vivienda.

Los subsidios federales vigentes hasta el 2018 que se destacan por aportación y frecuencia de uso son los siguientes — presentados en el Cuadro 1:

Cuadro 1. Subsidios federales vigentes hasta el 2018 que se destacan por aportación y frecuencia de uso.

\begin{tabular}{|c|c|c|c|}
\hline $\begin{array}{l}\text { Dependencia } \\
\text { Programa / Fondo }\end{array}$ & Objetivo específico & Población objetivo & Tipos de apoyo \\
\hline $\begin{array}{l}\text { CONAVI-SEDATU } \\
\text { Programa de } \\
\text { Acceso al } \\
\text { Financiamiento para } \\
\text { Soluciones } \\
\text { Habitacionales }\end{array}$ & $\begin{array}{l}\text { Ampliar el acceso al financiamiento de } \\
\text { la población de bajos ingresos para } \\
\text { soluciones habitacionales en un entorno } \\
\text { de crecimiento urbano ordenado y } \\
\text { sustentable }\end{array}$ & $\begin{array}{l}\text { Población de bajos ingresos con } \\
\text { necesidades de vivienda, que tienen } \\
\text { capacidad de obtener un } \\
\text { financiamiento y que pueden aportar } \\
\text { un ahorro previo }\end{array}$ & $\begin{array}{l}\text {-Adquisición de } \\
\text { vivienda, nueva o } \\
\text { usada } \\
\text {-Ampliación y/o } \\
\text { mejoramiento } \\
\text { - Adquisición de lote } \\
\text { con servicios } \\
\text {-Autoproducción de } \\
\text { vivienda }\end{array}$ \\
\hline
\end{tabular}

\footnotetext{
${ }^{6}$ Consultada en : <https://www.coneval.org.mx> el 31 de julio de 2018.
} 


\begin{tabular}{|c|c|c|c|}
\hline $\begin{array}{l}\text { FONHAPO- } \\
\text { SEDATU Programa } \\
\text { Apoyo a la Vivienda }\end{array}$ & $\begin{array}{l}\text { Mejorar las condiciones habitacionales } \\
\text { de los hogares mexicanos con ingresos } \\
\text { por debajo de la línea de bienestar y con } \\
\text { carencia por calidad y espacios de la } \\
\text { vivienda, con la finalidad de reducir el } \\
\text { rezago habitacional de los hogares } \\
\text { mexicanos }\end{array}$ & $\begin{array}{l}\text { Hogares mexicanos en localidades } \\
\text { urbanas y rurales con ingresos por } \\
\text { debajo de la línea de bienestar y con } \\
\text { carencia por calidad y espacios de la } \\
\text { vivienda, con especial atención a la } \\
\text { reubicación de aquellos que se } \\
\text { encuentren en zonas de alto riesgo o } \\
\text { que alguno de sus integrantes } \\
\text { pertenezca a los grupos sociales en } \\
\text { situación de vulnerabilidad }\end{array}$ & $\begin{array}{l}\text { - Construcción de } \\
\text { unidad básica de } \\
\text { vivienda (UBV) } \\
\text {-Ampliación } \\
\text { - Mejoramiento }\end{array}$ \\
\hline $\begin{array}{l}\text { SEDESOL Fondo } \\
\text { de Aportaciones } \\
\text { para la } \\
\text { Infraestructura } \\
\text { Social }\end{array}$ & $\begin{array}{l}\text { Mejorar la calidad de vida de las } \\
\text { familias en estado de pobreza y rezago } \\
\text { social, a través de ampliaciones a la } \\
\text { vivienda (cuarto, cocina o baño) y } \\
\text { mejoramientos (sustitución de techos, } \\
\text { muros o pisos precarios) }\end{array}$ & $\begin{array}{l}\text { Beneficiar directamente a población } \\
\text { en pobreza extrema y localidades con } \\
\text { alto o muy alto nivel de rezago social }\end{array}$ & $\begin{array}{l}\text {-Ampliación } \\
\text {-Mejoramiento }\end{array}$ \\
\hline $\begin{array}{l}\text { SEDATU Programa } \\
\text { de Infraestructura }\end{array}$ & $\begin{array}{l}\text { Mejorar la disponibilidad de la } \\
\text { infraestructura básica, complementaria, } \\
\text { además de dotar con equipamiento, } \\
\text { imagen, entorno, ampliación y } \\
\text { mejoramiento de la vivienda. Lo } \\
\text { anterior para mejorar las condiciones de } \\
\text { habitabilidad de los hogares que se } \\
\text { encuentran asentados en las Zonas de } \\
\text { Actuación del Programa }\end{array}$ & $\begin{array}{l}\text { Hogares que se encuentran asentados } \\
\text { en las Zonas de Actuación del } \\
\text { Programa, susceptibles de } \\
\text { intervención que presenten } \\
\text { deficientes condiciones de } \\
\text { habitabilidad y/o poca disponibilidad } \\
\text { de espacios habitables }\end{array}$ & $\begin{array}{l}\text {-Ampliación } \\
\text {-Mejoramiento }\end{array}$ \\
\hline
\end{tabular}

Los programas tienen diferentes lineamientos y reglas de operación que se ajustan periódicamente para mejorar el proceso de asignación del subsidio.

\section{Número de habitantes por vivienda}

La cantidad de personas que usan un departamento es un aspecto muy sensible, porque hay una relación directa entre el número de habitantes por vivienda y el número de cuartos de la misma.

Se considera, desde información emitida por la Secretaría de Desarrollo Agrario, Territorial y Urbano (SEDATU), que una vivienda presenta condiciones de hacinamiento si se supera el número de 2.5 habitantes por habitación (no se consideran espacios destinados a cocinar, baños o estancias).

Es relevante considerar el número de habitantes por vivienda social y el posible aumento de personas en la misma, ya que, además de los problemas asociados a la distribución de sus habitantes dentro de las habitaciones (potencia de hacinamiento), existen otro tipo de problemas sociales al compartir espacios para pernoctar entre individuos que no son necesariamente consanguíneos.

En los datos que presenta el Instituto Nacional de Estadística y Geografía (INEGI), en el Panorama Sociodemográfico de Aguascalientes, podemos observar que el promedio de habitantes por vivienda es de 3.9 (Figura 3) en la entidad, mientras que los pobladores del fraccionamiento de vivienda social vertical estudiado en la misma entidad, tiene una tercera parte de la ocupación de los departamentos con 5 personas o más, siendo 5 el número más recurrente de individuos por vivienda (Figura 4).

PANORAMA SOCIODEMOGRÁFICO DE AGUASCALIENTES 2015

(CENSO 2015 INEGI)

Total de viviendas particulaes habitadas

334589 Representa el 1.0\% del total nacional

Promedio de ocupantes po vivienda

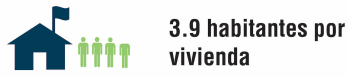

vivienda

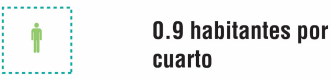

Figura 3. Panorama sociodemográfico de Aguascalientes 2015. 


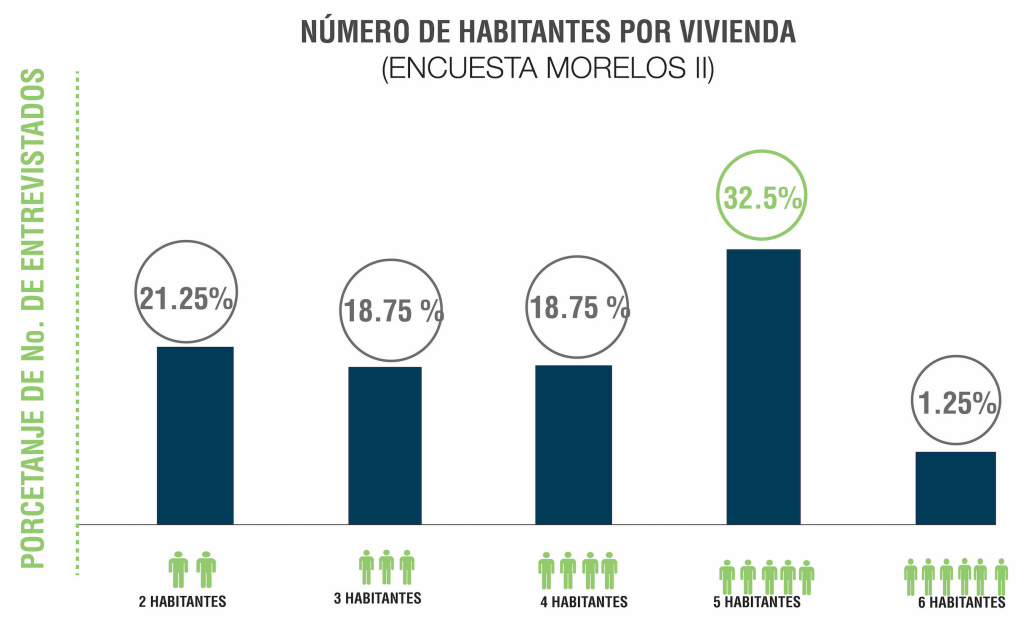

Figura 4. Número de habitantes por vivienda de fraccionamiento vivienda social en vertical.

Por lo anterior, y de acuerdo con los datos del CONEVAL, las unidades básicas de vivienda (UBV) con 2.5 o más habitantes por cuarto se encuentran en hacinamiento. Las viviendas del fraccionamiento estudiado tienen 2 recámaras por unidad, así que, los casos con más de 2.5 individuos por habitación, se encuentran en hacinamiento. Además, hay que considerar que, en muchas situaciones, duermen en el mismo cuarto personas adolescentes o adultas de diferente sexo que no son pareja. Lo anterior, tiene propensión a reducir privacidad entre otros factores dignos de ser examinados (y que salen del alcance del presente análisis).

Por lo tanto, una estrategia fundamental para ser considerada, es crear viviendas con la posibilidad de modificar espacios para ampliar el número de habitaciones a tres ${ }^{7}$.

\section{La forma en que se usa el espacio a partir de la disposición del mobiliario}

Si bien, puede percibirse, que el mobiliario dentro de una vivienda es el complemento que tiene que ser revisado por cada habitante, se considera fundamental que dentro del proyecto, se propongan diferentes alternativas como guías para el habitante, mejorar el desempeño espacial y el uso interior de la vivienda.

Normalmente, los habitantes de las viviendas sociales, no tienen la capacitación adecuada para diseñar un espacio. Por lo anterior, el acomodo espacial a partir del mobiliario, se convierte en un acto intuitivo, condicionado en muchas ocasiones por los muebles que poseía la familia antes de ocupar las viviendas sociales verticales.

Tomando en cuenta que el poder adquisitivo, para muchas de las familias que habitan en las viviendas sociales en vertical estudiadas, es limitado, es imperante que las propuestas arquitectónicas tengan un alto componente de flexibilidad para permitir que los diferentes perfiles de habitantes puedan acomodar su mobiliario y encontrar mejores formas de acomodo espacial con sus pertenencias.

Otro aspecto analizado dentro del estudio fue la cantidad de espacio libre comparado contra el utilizado por los muebles dispuestos. Los espacios analizados fueron: 1) el espacio de la cocina, sala y comedor y 2) en las habitaciones.

En las siguientes láminas (Figuras 5, 6, 7 y 8) se muestra el interior de las viviendas ocupadas y su referencia con un esquema de la planta arquitectónica para ver el acomodo, circulaciones y disposición del mobiliario de los espacios de cocina, sala y comedor.

Como se muestra en las láminas mencionadas con su referencia en las fotografías, en el acomodo de los muebles y su uso, son fundamentales para asegurar un adecuado orden espacial, ya que, los departamentos con muebles grandes o con mucho mobiliario, tienden a tener una circulación deficiente, además de complicar las diferentes funciones que se realizan dentro del departamento. Por el contrario, los departamentos con mobiliarios flexibles y con una mejor disposición, generan una percepción de mayor amplitud y facilitan las múltiples actividades que se realizan en los espacios.

\footnotetext{
${ }^{7}$ Se recomienda ver propuestas planteadas por el Ministerio de Vivienda y Urbanismo de Chile. Caso SERVIU Región de Santiago.
} 


\section{Labor \& Engenho}

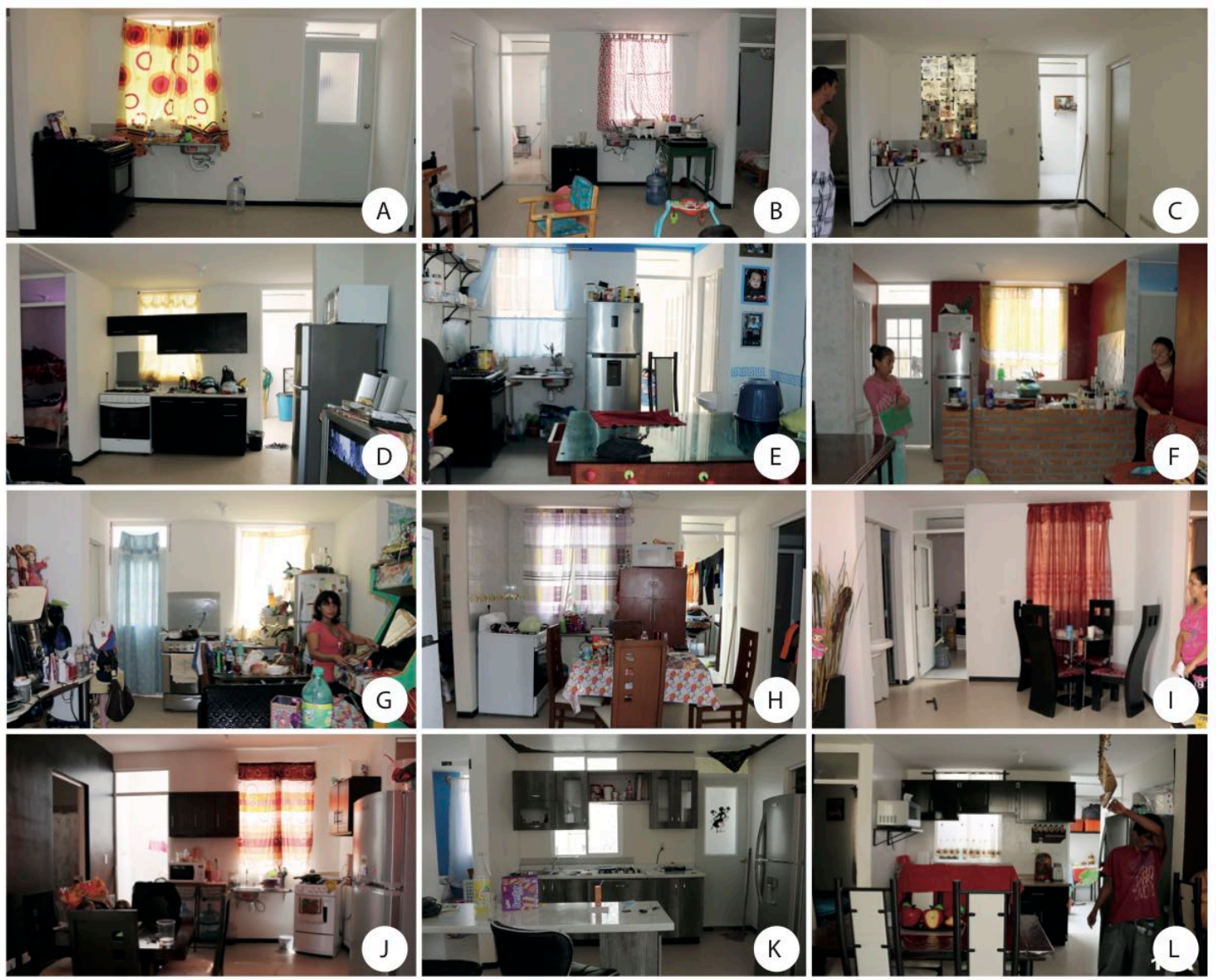

Figura 5. Tipo de ocupación espacial en función de mobiliario y acomodo de cocina y comedor.

Bañón

Figura 6. Tipo de ocupación espacial en función de mobiliario y acomodo de cocina y comedor en planta. 


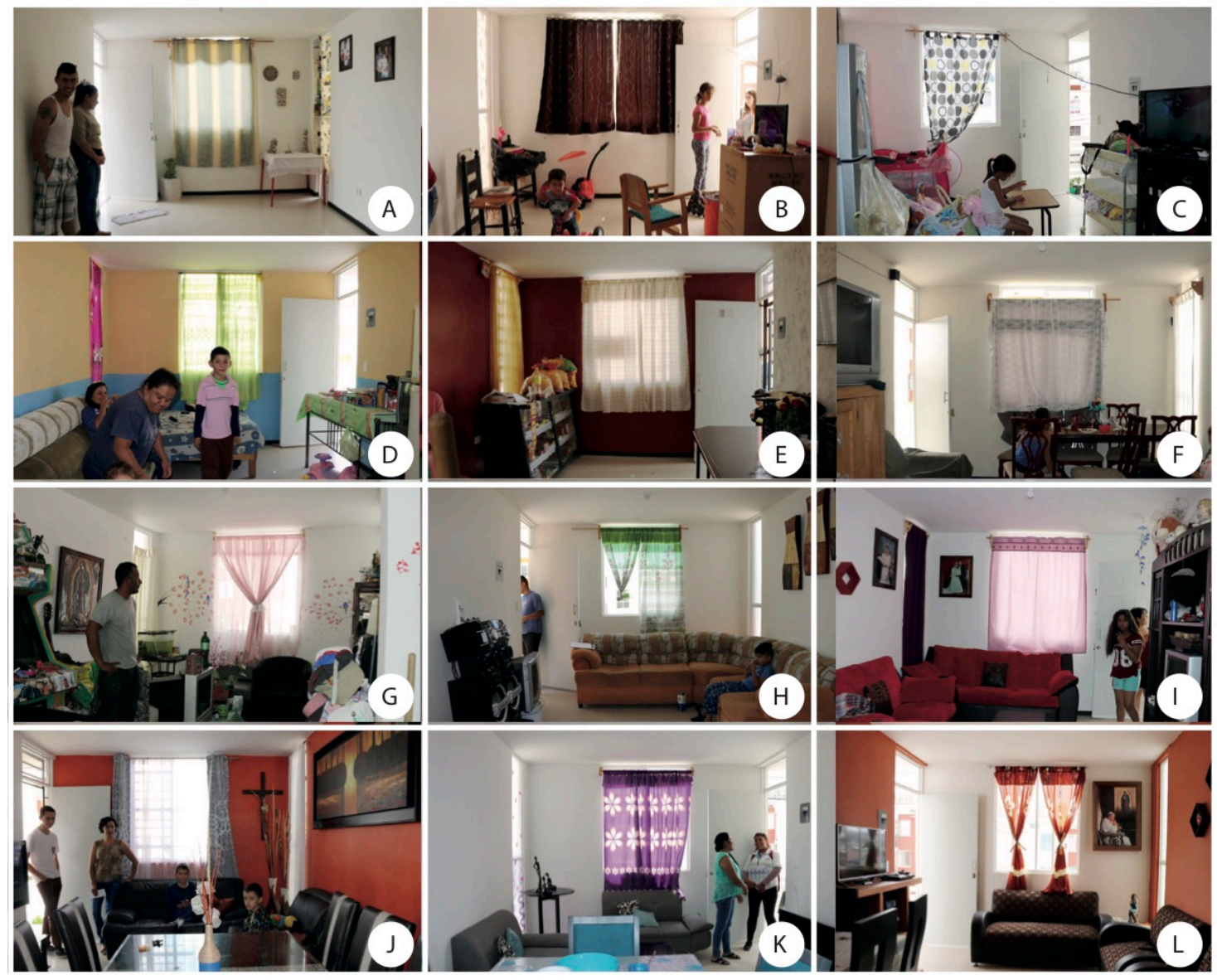

Figura 7. Tipo de ocupación espacial en función de mobiliario y acomodo de sala.

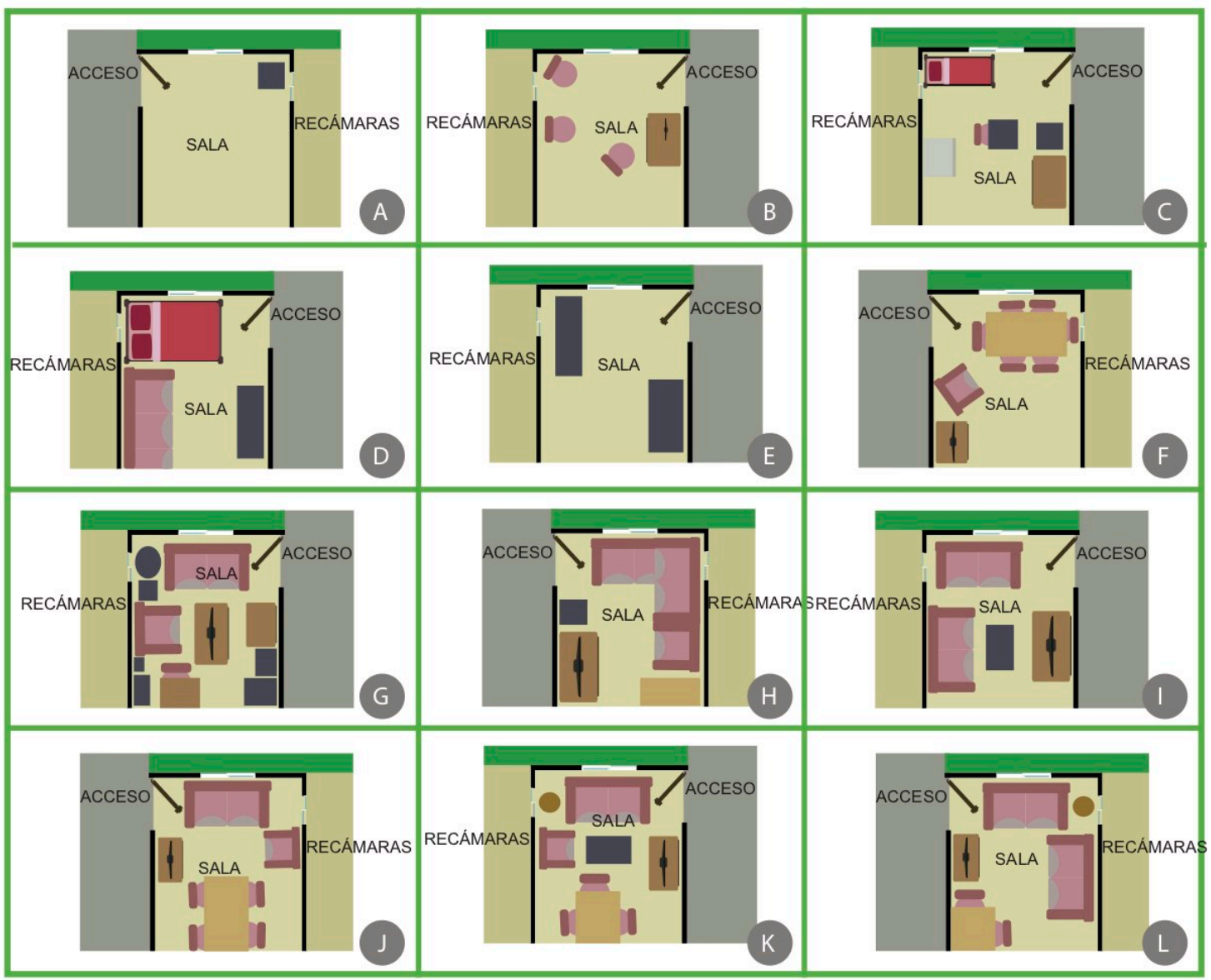

Figura 8. Tipo de ocupación espacial en función de mobiliario y acomodo sala en planta. 


\section{Preierencia de espacios para crecimiento de la vivienda por uso}

La capacidad de adaptabilidad de las viviendas, se relaciona con la potencia para poder encontrar más formas de usar el habitáculo de manera satisfactoria, al acomodar y cambiar disposiciones de la misma. Consecuentemente, uno de los aspectos dignos para ser incluidos dentro del diseño arquitectónico de las viviendas sociales, es la potencia para adaptarse en el tiempo de acuerdo con las condiciones evolutivas de la familia.

Para considerar aspectos adaptativos al diseño de una vivienda, es necesario conocer los principales aspectos de la edificación para ser adaptados por la familia. El estudio realizado en el fraccionamiento Morelos II, ha permitido conocer la preferencia de adaptación de los espacio, a partir del crecimiento del departamento por un uso específico, en el caso, en que la edificación tuviera la potencia para ampliar su tamaño, es decir, la potencia de adaptabilidad que debería de tener la vivienda social vertical.

En la encuesta realizada, considerando que la vivienda es un bien inmueble, patrimonio familiar, se preguntó al beneficiario lo siguiente: ¿cuál sería el espacio que debería de ampliarse (o ajustarse) en la vivienda, para resolver problemas, necesidades o deseos actuales o futuros?

La mayoría de los encuestados respondieron que debe de crecer la vivienda hacia el área de descanso, es decir, una habitación más (Figura 9). Lo anterior, tiene concordancia porque un número representativo de familias, el $41.2 \%$ del total de las viviendas encuestadas, tienen 5 o más habitantes, así que, necesitan un cuarto adicional para mejorar su calidad de vida.

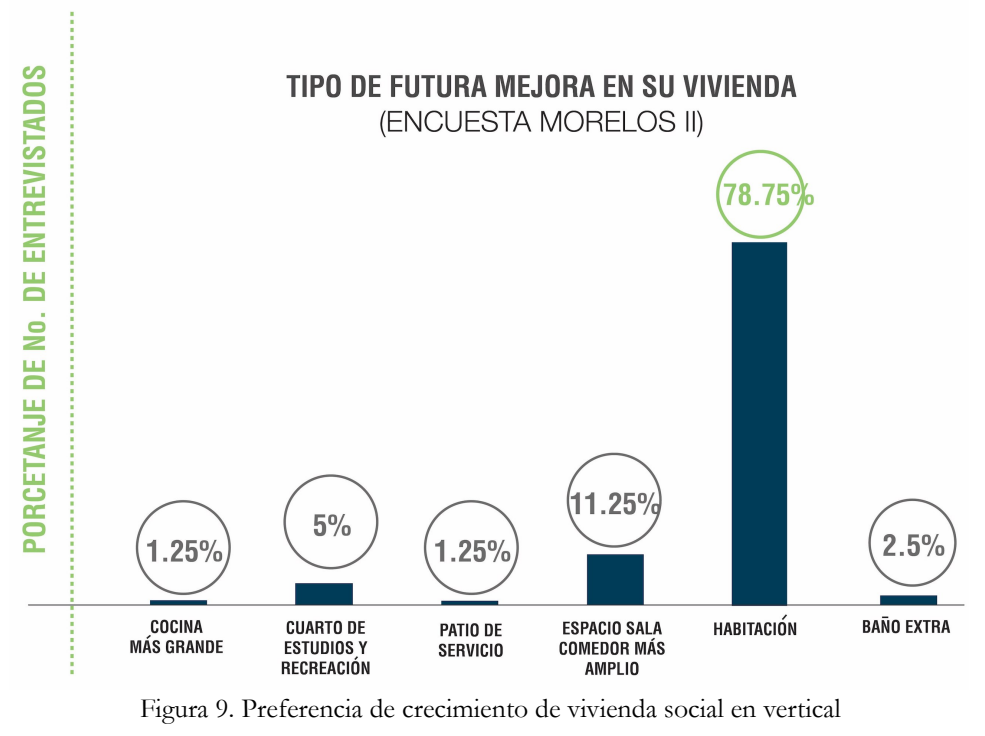

Es relevante mencionar que, de manera recurrente, los habitantes del fraccionamiento encuestado, mencionaron que hubieran preferido pagar menos por la vivienda al suprimir el estacionamiento. Lo anterior responde a que, la mayoría de los habitantes del fraccionamiento, no cuentan con automóvil propio. Su medio de transporte más recurrente es el público, así que, se enfatiza la necesidad de una buena ubicación para reducir los gastos familiares.

\section{Conclusiones}

Con la relación entre el ingreso y el gasto, de la mayoría de las familias con carencia patrimonial, se hace evidente que la planeación y el diseño adecuado en las edificaciones de vivienda social, son aspectos fundamentales para generar condiciones de acceso a la vivienda social a las familias en estado de pobreza y vulnerabilidad.

Los bajos ingresos de los solicitantes y los altos costos de las viviendas con servicios y equipamientos adecuados, reducen la posibilidad de que las familias en estado de pobreza y en vulnerabilidad, puedan acceder a la compra de una vivienda.

Los desarrolladores inmobiliarios necesitan vender altos volúmenes de edificaciones, así que se ha desarrollado un mercado muy lucrativo con el desarrollo de vivienda social. Para hacer más barato el costo de las viviendas, existe una tendencia a comprar terreno barato por parte de los desarrolladores. Sin embargo, la mayoría de estos terrenos no cuentan con los servicios necesarios, ni con las características 
básicas para ofrecer una calidad de vida adecuada para a los habitantes en términos de movilidad, seguridad servicios e infraestructura.

Por lo anterior, el incremento, utilización y distribución adecuada de todos los subsidios, es un componente fundamental para reducir el rezago habitacional en el país. Así, los involucrados, deberán de conocer y aplicar adecuadamente las reglas de operación y los lineamientos vigentes para el desarrollo de vivienda social.

El diseño arquitectónico y la disposición de los componentes para aumentar la adaptabilidad de los espacios dentro de una vivienda social, son características que ayudarán a los habitantes a poder vivir mejor. Ya sea para resolver un problema de hacinamiento o de género, la adaptación de una nueva habitación puede mejorar notoriamente la calidad de vida de los habitantes de las viviendas sociales.

Así, tanto el programa arquitectónico, como los códigos regionales vigentes para la edificación, tienen que ser revisados, ya que, en muchas ocasiones, se solicitan características y espacios poco útiles para el habitante de un sector determinado, como es el estacionamiento para la mayoría de los habitantes que no tienen coche.

Con las consideraciones mencionadas, se pretende mostrar un punto de reflexión para que los involucrados en el desarrollo de vivienda social en vertical, puedan considerar soluciones, deficiencias o características con gran impacto en los habitantes de las viviendas sociales. Así, los aspectos evaluados, son sólo algunos de los que pueden tener relevancia en los diferentes proyectos por las mismas condiciones con las que se genera cada propuesta.

Siguiendo la línea de la pregunta del planteamiento inicial, el significado de una casa, debería de comenzar por ser la plataforma adecuada para desarrollar el hábitat de los individuos en sociedad, es decir, el lugar adecuado en donde se realizan las prácticas sociales y a través de donde se reconstruye la interpretación de la sociedad. Así, cada desarrollador de vivienda social, debe de tener el compromiso de dar respuestas adecuadas y de impulsar una aspiración para aumentar la calidad de vida de los habitantes de sus desarrollos, ya que la vivienda, más que un contenedor, es la plataforma para desarrollar una comunidad plena.

\section{Reierencias}

Bauman, Z. (1999). Trabajo, consumismo y nuevos pobres. Barcelona [España]: Editorial Gedisa.

Bauman, Z. (2005). Vidas desperdiciadas. La modernidad y sus parias. Barcelona [España]: Editorial Paidós.

Bauman, Z. (2008). Comunidad. Madrid [España]: Editorial Siglo XXI.

Bauman, Z. (2013). La vida líquida. Barcelona [España]: Editorial Planeta.

Boff, L. (2001). Ética planetaria desde el Gran Sur. Madrid [España]: Editorial Trotta.

Brebbia, C. y Pulselli, \& R. (editores). (2014). Eco-Architecture V. Reino Unido: Editorial Wessex Institute of Technology.

CONEVAL. (2018). Línea de bienestar 2018. 31 de julio de 2018, de CONEVAL. Recuperado de: < http:// www.coneval.gob.mx >

Consejo Nacional de Población (CONAPO). Recuperado de: <http://www.conapo.gob.mx >

Drew, P. (2001). Touch This Earth Lightly. Glenn Murcutt in his own words. Sydney [Australia]: Duffy \& Snellgrove.

Eco, U. (1984). Obra abierta. Barcelona [España]: Editorial Lumen.

Eco, U (1998). El superhombre de masas. Barcelona [España]: Editorial Lumen.

Eco, U (1999). La estructura ausente. Introducción a la semiótica. Barcelona [España]: Editorial Lumen.

Hardt, M., y Negri, A. (2000). Empire. Cambridge [Massachussets, E.U.A.]: Harvard University Press.

Higueras, E. (2006). Urbanismo Bioclimático. Barcelona [España]: Gustavo Gili.

Instituto Nacional de Estadística y Geografía (INEGI), Encuesta Nacional de Ocupación y Empleo (ENOE), 2011. Recuperado de: <http: www.inegi.org.mx>. Encuesta Nacional de Población, 2000, 2010. 
Irigoyen, J. (1998). Filosofía y diseño. Una aproximación epistemológica. México [D.F., México]: Editorial Universidad Autónoma Metropolitana.

Lanzagorta, J. (2007). ADN de la arquitectura. Ética, significado y utilidad. Jalisco [México]: Editorial Pandora. Cuadernos de Trabajo ITESO.

Latouche, S. (2012). Salir de la sociedad de consumo. Voces y vías del decrecimiento. España: Octaedro.

Latouche, S. (2009) Decrecimiento y Posdesarrollo: El pensamiento creativo contra la economía del absurdo. Mataró [España]: Intervención Cultural.

Max-Neef, M., Elizalde, A., Hopenhayan, M. (1993). Desarrollo a escala bumana. Una opción para el futuro. Santiago [Chile]: Centro de Alternativas de Desarrollo, CEPAUR.

Morin, E. (1999). Los siete saberes necesarios para la educación del futuro. París [Francia]: UNESCO.

Pesci, R. (2007). Ambitectura: hacia un tratado de arquitectura, ciudad y ambiente. Argentina: Al Margen.

Rapaille, C. (2007). El código cultural. México [D.F., México]: Grupo Editorial Norma.

Ricard, A. (1982). Diseño. ¿Por qué?. Barcelona [España]: Editorial Gustavo Gili.

Richardson, P. (2007). XS ecológico. Grandes ideas para pequeños edificios. Barcelona [España]: Editorial Gustavo Gili.

Rogers, R. (2001). Ciudades para un pequeño planeta. Barcelona [España]: Editorial Gustavo Gili.

Sartelli, E. (2014). La cajita infeliz. Un viaje a través de la sociedad capitalista. España: Akal.

Safdie, M. (1996). The city alter the automobile. An Architect's Vision. Chicago [E.U.A.]: Editorial Stoddart.

Sen, A. (2000). Desarrollo y Libertad. Argentina: Editorial Planeta.

Sinclair, C. y Stohr, K. (2006). Design like you give a damn. Architectural Responses to humanitarian crises, E.U.A.: Metropolis Books.

Stiglitz, J. (2002). El malestar de la Globalización. Madrid [España]: Editorial Punto de lectura.

Stiglitz, J. (2012). El precio de la desigualdad. Madrid [España]: Editorial Taurus.

WWF Internacional (World Wide Fund for Nature ) (2014). Informe Planeta Vivo 2014. Madrid [España]: World Wide Fund for Nature.

Yeang, K.(2006) Ecodesign. A manual for Ecological Design. Londres [Reino Unido]: Wiley Academy. 